\title{
Voltage Magnitude State Estimation By ANNs with Reduction of PMUs
}

\author{
M. Gholami ${ }^{1}$, G. B. Gharehpetian ${ }^{1}$, B. Fahimi ${ }^{2}$ and M. J. Sanjari ${ }^{1}$ \\ 1 Electrical Engineering Department, Amirkabir University of Technology, Tehran. Iran \\ Phone/Fax number: +0098 2164543504, \\ E-mail: mostafagholami@aut.ac.ir, grptian@aut.ac.ir, $\underline{\text { m_j_sanjari@aut.ac.ir, }}$ \\ 2 Electrical Engineering Department, University of Texas at Dallas \\ E-mail: fahimi@uta.edu
}

\begin{abstract}
In this paper, the relationship between the number of installed PMUs and the accuracy of the monitoring system has been studied. In the best case, all buses are observable. In this paper, it is assumed that the economical saving or failure of PMUs can result in unobservable buses. In this case, Artificial Neural Network (ANN) has been used to estimate the voltage of unobservable buses. The proposed method has been applied to IEEE 14-Bus system. Based on single contingencies analysis, it is shown that in small scale networks, such as IEEE 14-Bus system, the ANN can estimate the unobservable buses with neglectable error.
\end{abstract}

\section{Key words}

State Estimation, PMU, Incomplete Observability, Artificial Neural Network.

\section{Introduction}

PMUs can get the magnitude and phase of voltage of the bus that is connected to, and the magnitude and phase of current of lines that are connected to that bus. One of the important questions related to PMUs is the determination of the minimum number of required PMUs, which should be installed in the power system, because they are expensive devices and cannot be installed on all buses. In [1], it is shown that the PMU placement is a NP-Complete (Nondeterministic Polynomial time) problem and there is no closed form solution for it.

In [2] and [8], an algorithm based on Integer Linear Programming (ILP) has been presented to find the minimum number of PMUs that are required to make all grid buses observable. In [2], an algorithm has been presented for multistage installation of PMUs, too. This problem has been solved by Binary ILP (BILP) [3] and [6]. In [4], the concept of unobservability has been studied and a solution for the PMU placement problem has been presented.

In [7], a virtual data elimination preprocessing method and a matrix reduction algorithm has been suggested, to reduce the scale of a placement problem.

In [9], [10] and [11], the state estimation problem has been solved for PMUs. The PMU can measure voltage phasors of the PMU node and the current phasors of the branches, which are connected to this node. So, state variables of these nodes are known.

In this paper, the main object is the reduction of PMU installation cost, by the reduction of the number of installed PMUs. After applying this strategy, some of network buses will be unobservable. As a result, this strategy has negative effects on monitoring or efficient control of the power system. To overcome this problem, unobservable buses voltages must be estimated. The reduction of installed PMUs decreases the accuracy of the monitoring system. As a result, this strategy can be used, when the maximum error of the estimation of system states is small enough and negligible. In this paper, ANN (Artificial Neural Network) has been used to estimate voltages of unobservable buses. It should be noted that the proposed method can be used for the case when one PMU has failed or its communication system has interrupted.

\section{Estimation by ANN}

In this paper, PMUs will be optimally placed considering two important points; the first point is the installation cost of PMUs and the second one is the error of the estimation. If a power system needs an accurate monitoring system, it should have enough installed PMUs. The reduction of the number of PMUs results in unobservable buses. In this paper, the ANN has been used to estimate the voltages of unobservable buses. The power network has been simulated by DIgSILENT software and the ANN has been carried out in MATLAB software. As it is mentioned before, this idea can be used when there is complete observability but one of PMUs or its communication system has failed. From this point of view, the ANN related to each PMU has been trained, and when one PMU fails, the ANN related to that PMU can be used and the voltages of buses, which are unobservable can be estimated by ANN.

The ANN should be trained by the results of the load flow analysis. The scenarios have been randomly selected for different load or generation levels, which are generated by using Mont-Carlo method. In this method, a random number $(N)$ between 1 and $N_{b}$ (the 
number of buses) is generated. This number determines the number of buses, which their parameters should be changed. If a bus is a PQ or PV bus, then in order to change its parameters, scenario 1 or 2 should be selected, respectively.

In the scenario 1, the load active power is randomly selected in the prespecified margin, as follows:

$$
P_{L, i}^{P Q} \in\left[P_{L, i}^{\min } \quad P_{L, i}^{\max }\right]
$$

In the scenario 2, one of the following three cases, is selected with equal probability, (i.e., 1/3):

Case 1: Only the load active power should be changed, as follows:

$$
P_{L, i}^{P V} \in\left[P_{L, i}^{\min } \quad P_{L, i}^{\max }\right]
$$

Case 2: Only the active power generation should be changed, as follows:

$$
P_{G, i}^{P V} \in\left[\begin{array}{ll}
P_{G, i}^{\min } & P_{G, i}^{\max }
\end{array}\right]
$$

Case 3: Both, the bus load and generation should be changed by using the following constraints:

$$
\begin{aligned}
& P_{L, i}^{P V} \in\left[\begin{array}{ll}
P_{L, i}^{\min } & P_{L, i}^{\max }
\end{array}\right] \\
& \text { and } \\
& P_{G, i}^{P V} \in\left[\begin{array}{ll}
P_{G, i}^{\min } & P_{G, i}^{\max }
\end{array}\right]
\end{aligned}
$$

The ANN used in this paper has 13 nodes on the input and one hidden layer with 20 nodes. The Levenberg-Marquart back-propagation algorithm has been used to identify ANN parameters.

In this step, 1000 different load flow scenarios have been simulated by DIgSILENT. 900 of them have been selected to train the ANN, and 100 scenarios have been chosen to test the ANN. The error has been calculated for these 100 scenarios by the following equation:

$$
\operatorname{Error}(\%)=\frac{\text { Actual Value }- \text { Estimated Value }}{\text { Actual Value }} * 100
$$

The maximum and the average values of error are calculated, to compare the different cases.

\section{Installed PMU Number Reduction}

In this section, the proposed algorithm has been applied to IEEE 14-Bus network.

\section{A. IEEE 14-BUS Test System}

It is shown in [5] that the IEEE 14-Bus system is observable by using 4 PMUs. To model the PMU failure, one of the PMUs should not be used. The candidate PMU has been selected by using the proposed index, named SORI. If the bus $i$ is observed by $n_{i}$ numbers of PMUs, then System Observability Redundancy Index (SORI) is expressed by the following equation:

$$
S O R I=\sum n_{i}
$$

Based on this index, the PMU installed on bus 7 should be considered as a failed PMU. In this case, the bus 8 will be unobservable. But, the voltage of this bus can be estimated by the trained ANN.

Based on SORI index, the next candidate buses can be selected and listed in Table-1.

Table-I: Eliminated PMUs in IEEE 14-Bus system

\begin{tabular}{|c|c|c|}
\hline $\begin{array}{c}\text { No. of } \\
\text { eliminated } \\
\text { PMUs }\end{array}$ & No. of PMUs & $\begin{array}{c}\text { Installed on } \\
\text { buses }\end{array}$ \\
\hline 0 & 4 & $2,6,7$ and 9 \\
\hline 1 & 3 & 2,6 and 9 \\
\hline 2 & 2 & 2 and 6 \\
\hline
\end{tabular}

The error of the estimation of the bus 8 voltage (with 3 PMUs) is given in Table II.

Table-II: Error of estimation of voltage of bus 8 (by using 3 PMUs).

\begin{tabular}{|l|l|}
\hline & Error \% \\
\hline Maximum Error & 0.0028 \\
\hline Average Error & $2.46 * 10^{-5}$ \\
\hline
\end{tabular}

The errors represent the difference between the results calculated by ANN and simulated by DIgSILENT. Considering the Table-II, it is obvious that the reduction of one PMU leads to a negligible error. Based on the results given in Table-I, it is possible to eliminate the second PMU at bus 9. The error between the estimated value and the real value for this case has been represented in Table-III.

Table-III: Error of estimation of voltages of unobservable

\begin{tabular}{|l|c|}
\multicolumn{1}{c}{ buses (by using 2 PMUs). } \\
\hline Maximum Error & 0.73 \\
\hline Average Error & 0.18 \\
\hline
\end{tabular}

The relationship between the number of PMUs and the error between the estimated and real values has been depicted in Fig-2. The relationship between the number of PMUs and the cost of the monitoring system is shown in Fig-3. As shown in Fig-2 and Fig3 , the less the error, the more the cost. 


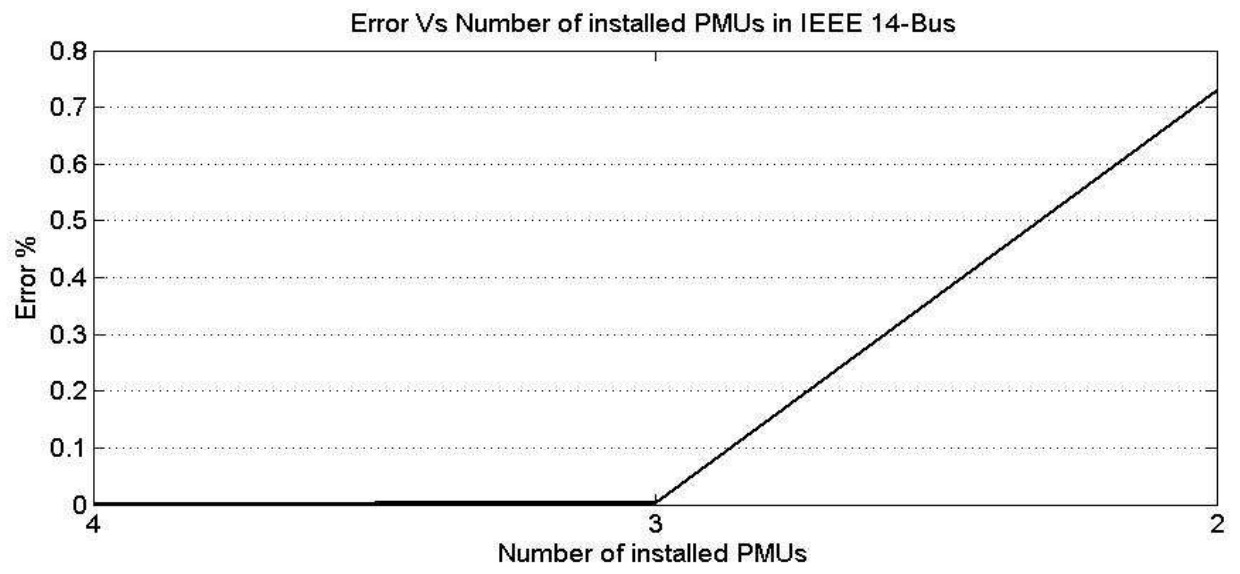

Fig-1: Error versus number of installed PMUs in IEEE 14-Bus system Cost of Monitoring System Vs Number of installed PMUs in IEEE 14-Bus

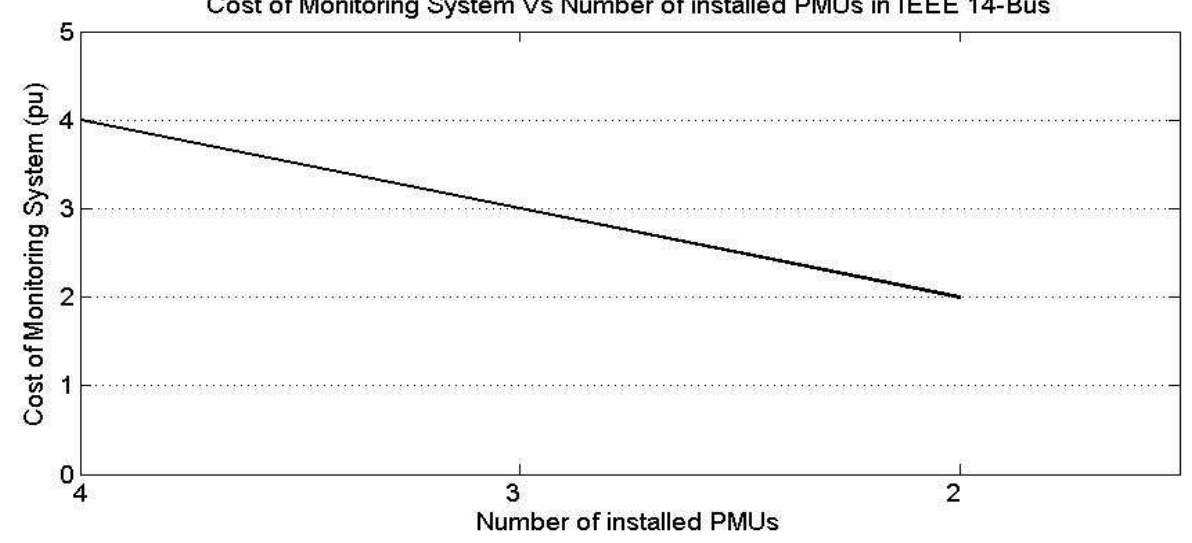

Fig-2: Cost of monitoring system versus number of installed PMUs in IEEE 14-Bus system

As shown in Fig-1 and Fig-2, decreasing the number of installed PMUs leads to increasing the error of estimation and decreasing the cost of monitoring system. In other word, there is a trade-off between the error of the estimation and the cost of the monitoring system. Therefore, considering an acceptable range of error, it is possible to reduce the number of installed PMUs.

\section{Single Contingency Studies}

In this section, it is assumed that the voltage of buses should be estimated by the proposed algorithm after occurrence of a single contingency. The IEEE 14-bus has been studied and the results have been given in Table IV.

Table-IV: Maximum error of voltage estimation of unobservable buses after occurrence of contingency in

\begin{tabular}{|c|c|c|}
\hline $\begin{array}{c}\text { No. of } \\
\text { installed PMUs }\end{array}$ & $\begin{array}{c}\text { Maximum } \\
\text { Error } \%\end{array}$ & $\begin{array}{c}\text { Average } \\
\text { Error } \%\end{array}$ \\
\hline 4 & 0 & 0 \\
\hline 3 & 21.84 & 6.57 \\
\hline 2 & 8.42 & 3.56 \\
\hline
\end{tabular}

It can be seen in this table that the errors are considerable and cannot be neglected. The main cause of this problem is the radial configuration of some parts of the network. In this case, the outage of one line can result in subnetworks without any generation (passive network). Therefore, the voltage of some buses are equal to zero.

Training with these type of data increase the nonlinearity of the problem and the wrong estimation probability by ANN.

This problem can be solved in a smart grid, which has sensors, like voltage transformers, for the detection of the outage of this type of transmission lines.

IEEE 14-bus test system has only one line with this condition, which is the line between bus 7 and bus 8 . If a sensor, which can detect the line outage, would be installed on this line, then the results could be changed, as given in Table- $\mathrm{V}$. 
Table-V: Maximum error of voltage estimation of unobservable buses in IEEE 14-Bus smart grid

\begin{tabular}{|c|c|c|}
\hline $\begin{array}{c}\text { No. of } \\
\text { installed PMUs }\end{array}$ & $\begin{array}{c}\text { Maximum } \\
\text { Error \% }\end{array}$ & $\begin{array}{c}\text { Average } \\
\text { Error \% }\end{array}$ \\
\hline 4 & 0 & 0 \\
\hline 3 & 0.019 & 0.0035 \\
\hline 2 & 2.46 & 0.15 \\
\hline
\end{tabular}

\section{Conclusion}

In this paper it has been shown that the reduction of installed PMUs and the application of ANNs for the estimation of unobservable buses increases errors in voltage estimation, but decreases the cost of monitoring system. The system designer should find a trade-off between error and cost.

The proposed algorithm has been applied to IEEE 14Bus test system. It is shown that for normal operation conditions, it is possible to reduce the number of installed PMUs, if there is an acceptable error range. But in case of single contingency, this solution do not has a good performance and the errors are unacceptable. By using voltage sensors, it is possible to change the test systems to smart grids. The voltages of unobservable buses of IEEE 14-Bus smart system can be estimated by ANNs with a good performance.

\section{References}

[1] D. J. Brueni and L. S. Heath, "The PMU Placement Problem," SIAM J.Discrete Math., vol. 19, no. 3, pp. 744-761, Dec. 2005.

[2] D. Dua, S. Dambhare, R. K. Gajbhiye, and S. A. Soman, "Optimal Multistage Scheduling of PMUPlacement: An ILP Approach" IEEE Trans.onPower Delivery, Vol. 23, No. 4, Oct. 2008
[3] N. H. Abbasy and H. M. Ismail, "A Unified Approach for the Optimal PMU Location for Power System State Estimation" IEEE Transon Power Systems, Vol. 24, No. 2, May 2009

[4] R. F. Nuqui and A. G. Phadke, "Phasor Measurement Unit Placement Techniques for Complete and Incomplete Observability" IEEE Trans.on Power Delivery, Vol. 20, No. 4, Oct. 2005

[5] A. G. Phadke, "Synchronisedphasor measurements in power systems," IEEE Comput. Applicat. Power, Vol. 6, No. 2, pp. 10-15, Apr. 1993.

[6] S. Chakrabarti and E. Kyriakides, "Optimal Placement of Phasor Measurement Units for Power System Observability" IEEE Trans. On Power Systems, Vol. 23, No. 3, August 2008.

[7] M. Zhou, V. A. Centeno, A. G. Phadke, Y. Hu, D. Noosel, H. A. R. Volskis, "A Preprocessing Method for Effective PMU Placement Studies", DRPT2008, Nanjing China.

[8] B. Gou, "Generalized Integer Linear Programming Formulation for Optimal PMU Placement", IEEE Trans. on Power Systems, Vol. 23, No. 3, August 2008.

[9] H. Zhao, "A New State Estimation Model of Utilizing PMU Measurements" International Conference on Power System Technology, India, 2006.

[10] A. Z. Gmm, I. N. Kolosok, A. M. Glazunova and E.S. Korkina, "PMU Plaement Criteria for EPS State Estimation" DRPT2008, Nanjing, China.

[11] F. Chen, X. Han, Z. Pan and L. Han, "State Estimation Model and Algorithm Including PMU", DRPT2008, Nanjing, China, 2008. 